\title{
A PRINCIPLE OF PREFERENCE CONCENTRATION APPLIED TO THE UNSUPERVISED EVALUATION OF THE IMPORTANCE OF MULTIPLE CRITERIA
}

\author{
Annibal Parracho SantAnna ${ }^{1 *}$ and Joana Lezan Sant' Anna ${ }^{2}$
}

Received May 05, 2018 / Accepted June 06, 2019

\begin{abstract}
The influence of a principle of preference concentration in the process of decision making in different contexts is discussed here. A procedure exploring the concentration of preferences to derive probabilities and another to derive, with higher generality, Choquet capacities are presented. An important feature of this last procedure is that it derives capacities in a space of criteria only from matrices of probabilities of preference according to each criterion, precluding the effort of directly trying to measure the interactions between them.
\end{abstract}

Keywords: multiple criteria analysis, preferences, Choquet integral.

\section{INTRODUCTION}

In multicriteria decision analysis, the decision alternatives are represented by vectors of evaluations according to different criteria. The global evaluation of each alternative by the multiple criteria is given by a real function of such vector, most frequently a linear function (Fishburn, 1970; Keeney \& Raiffa, 1976).

A probabilistic character is present in the preference assessments according to each criterion, due, for instance, to subjective factors that lead decision makers to assign in successive instants different meanings to the same attribute or to measurement errors that affect the evaluations. In a probabilistic view, the preferences according to each criterion are given by probabilities of choice and the linear combination may be considered as the combination of probabilities of being preferred according to each criterion conditional on the preference for such criterion. The weights of the linear combination are, according to this probabilistic view, the probabilities of each criterion being chosen.

\footnotetext{
*Corresponding author - https://orcid.org/0000-0001-6336-1688

${ }^{1}$ Programa de Doutorado em Sistemas de Gestão Sustentáveis, UFF, Niterói-RJ, Brazil. E-mail: annibal.parracho@gmail.com

${ }^{2}$ Programa de Pós-Graduação em Psicologia Social, Instituto de Psicologia, UERJ, Rio de Janeiro-RJ, Brazil.
} 
A practical problem in this criteria composition approach is: how to determine such weights? There are different techniques designed to deal with this problem. Two key ideas to empirically obtain vectors of weights are developed in Saaty (1980) and Simos (1974). For more details on other approaches, see also Hammond et al. (2015).

But this problem becomes much more complicated when redundancies and reciprocal influences between different criteria have to be taken into account. There are statistical approaches to, besides collecting information about the probabilities of preference according to the criteria and about the importance of the criteria, measure directly the inner relations between the sets of criteria (Grabisch et al., 2008; Angilella et al., 2015; Eyvindson et al., 2015; Benabbou et al., 2017; Meyer \& Olteanu, 2017).

Here, a different approach is taken, based on deducing probabilities of preference among the criteria, and, more generally, capacities for the subsets of criteria, from the matrix of probabilities of preference for each alternative according to each criterion. This line of inference, besides simplifying the decision process, avoids difficulties and distortions inherent in the direct evaluation of the highly abstract preferences for criteria.

To do that a principle of preference concentration, which drives the preference of the decision makers to those criteria ablest to point a best alternative, is here explored. According to this psychological principle, the importance of the criteria is determined by their power of concentrating the preference on a unique alternative.

This principle is implicitly applied in the use of the probabilities of being the best according to each criterion (Sant'Anna, 2010, 2015; Garcia \& Sant'Anna, 2015) in the Composition of Probabilistic Preferences (CPP). The use of the probabilities of being the best, obtained computing the probabilities of each alternative being preferred to all the others, induces a concentration by leading to a relative increase in the scores of those alternatives presenting the initially highest scores.

The next application of the principle consists in linearly deriving the vector of weights for the individual criteria from the vector of largest preferences that each of them is able to assign to its preferred alternative.

In a more general approach, allowing for the possibility of interactions, capacities (Choquet, 1953; Sugeno, 1974) substitute for probabilities. Then, the capacity of each set of criteria will be proportional to the largest probability of preference of an alternative by some criterion of the set. By this way, while the capacity of any set of two or more criteria increases with the convergence between them about which is the best alternative, the capacity of isolate criteria still increases with the probability that it assigns to its most preferred alternative.

Extracting information on preferences for criteria from available information about preferences for alternatives according to the criteria, besides opening new possibilities, dwells on a long tradition that may be related to the Bayes principle. In fact, this approach, even though not directly applying the Bayes rule, obtains information about the parameters of the distribution of prefer- 
ences among the criteria by combining new evidence to prior information. The new evidence is given by the evaluations of the different alternatives by the different criteria. The prior information is not given by an initial distribution of preferences among the criteria but by a preliminary rule for the composition of preferences by the multiple criteria.

To complete the analogy, the preference concentration principle employed to perform the transition in the multicriteria framework mirrors the maximum likelihood estimation rule by selecting that distribution of probabilities (or that capacity) that maximizes the preference for the most preferred alternative.

This appropriation of the Bayes Principle in the process of determining preferences among the criteria may be also observed in the Analytic Hierarchy Process (AHP) and Analytic Network Process (ANP) approaches (Saaty, 1980, 1996; Saaty \& Brady, 2009). In these methodologies for multiple criterion analysis, the evaluation of the alternatives by the criteria contributes in the weighting of the criteria (Mimović et al., 2015; Moreno-Jiménez et al., 2016) as well. A more direct application of the Bayes rule to recalculate the weights in multiple criteria decision analysis has been proposed by Vinogradova et al. (2018).

The transformation of the evaluations into probabilities of being the most preferred alternative brings all evaluations to the same scale of measurement. This property, of commensurability of the criteria, is an essential condition to make possible the combination by the Choquet integral. New approaches to combine preferences taking into account interactions between criteria, like those of Khrennikova \& Haven (2017) or Labreuche \& Grabisch (2013), the first using extensions of quantum probabilities (Born, 1954; Plotnitsky, 2009), and the second using generalized additive independence (GAI) (Fishburn, 1967; Bacchus \& Grove, 1995), may also benefit from this previous probabilistic transformation.

Another advantage of the transformation into probabilities of being the most preferred alternative derives from the reduction of the scores of alternatives that are not located close to the frontier of best alternatives. Composition methodologies like, for instance, those employing the Technique for Order of Preference by Similarity to Ideal Solution (TOPSIS) approach (Hwang \& Yoon, 1981; Kong, 2011), which linearly measure the distances to the frontiers, are more subject to the effect of the addition of new alternatives to the set of alternatives being compared (Lootsma, 1996; May et al., 2013). An ample review of this problem, of the possibility of rank reversal due to changes in the set of alternatives compared, has been recently provided by Aires \& Ferreira (2018). For a new discussion of the problem of determining the decision alternatives, see also Colorni \& Tsoukiàs (2018).

Throughout this paper, Section 2 discusses the principle of preference concentration and details the algorithms employed to build probabilities and capacities applying this principle. Section 3 discusses examples of its application in different contexts. Section 4 brings final comments. 


\section{THE PRINCIPLE OF PREFERENCE CONCENTRATION}

Different processes drive human decisions in different contexts and identifying principles applied to elicit preference from uncertain evidence may improve the knowledge about these processes (Luce, 1959; Camerer, 1987; McFadden et al., 1999). More recently, Kojadinovic (2004) and Rowley et al. (2015) addressed this subject in the context of combining, by means of a Choquet integral with respect to a capacity, multiple criteria that interact. An essential condition to make possible the combination by the Choquet integral is the commensurability of the criteria. This condition is granted if the preferences are determined by probabilities.

As in Kojadinovic (2004) and Rowley et al. (2015), the algorithms here developed to determine the capacity of the criteria are based only on the results of the application of these criteria to a set of possible alternatives. Instead of searching for outer information about the interaction, this approach leaves, in the pictorial word of Doyle (1995), the data "speak", free of any extrinsic influence.

The construction of the capacity explores the presence of a principle preference concentration driving the application of the criteria. This principle, consistent with the aversion to uncertainty in the decision process, leads the decision maker to seek the maximization of the ability to discriminate the most preferred alternative. This drives attention to the highest probabilities of choice according to each criterion.

The search for maximization of the distinction of the preferred alternative implies that a criterion or a set of criteria is more important (Grabisch, 1996) for the decision maker the more it is able to point out, from the alternatives among which the choice is processed, one alternative as the preferred one. This corresponds to the belief that, prevented or corrected possible blunders, high evaluations provide more reliable information about the decision priorities than the measurements of lower priorities.

This principle may be grounded on a psychological need of simplifying decisions (Gilovich et al., 2002; Dane \& Pratt, 2007). But it may have a more general basis. It may be derived from the disguised presence of determinant factors behind the criteria. There is no explanation for searching a best choice applying the criteria if they do not mirror factors that interact to determine a really best choice. These determinant factors combine in a simpler way to determine the best choice that the decision maker tries to discover digging the observed measurements.

Following this strategy, if a criterion has a clear preference for an alternative, then it will receive a high capacity as a unitary set. Also, if two criteria agree to give high preference for an alternative, even if it is not the most preferred by either of them, their high joint preference for this alternative assigns high capacity to the union of the two unitary sets. And so on, to combine more criteria.

Even when, in the evaluation of subsets of alternatives with low probability of being preferred two criteria point in different directions, if both agree about the alternatives with high probability of being the best, they positively interact in the decision, and their joint information should be given greater importance than that provided by each of them separately. The principle of 
preference concentration increases the measure of the interaction between them because the joint information provided increases the conviction of the decision maker concerned with determining the best alternative. If the contrary happens, we have a negative interaction.

In synthesis, there is positive interaction in the formation of preferences in the case of an agreement between the criteria with respect to which is the best choice and negative interaction in the case of disagreement.

\subsection{Interaction and statistical dependence}

Interaction has the effect of increasing or decreasing the value that the preference according to a given criterion assigns to other criteria. Thus, interaction is related to the personal aim of the decision maker to choose the best among the available alternatives. On the other hand, statistical dependence is related to the practical processing of this design. If information about dependence is available, it may be used in the process of determining the preference for each alternative according to subsets of criteria, while information about interaction is used to model the relations between the criteria.

There is interaction between two criteria when the preference for an alternative by one of them increases or reduces the importance of the preference for this or any other alternative according to the other. The same applies to groups of criteria, no matter the reason for this influence. Exchangeability, complementarity, substitutability or preferential dependence as defined, for instance, in Marichal (2000), may be such reasons. In this sense is that the presence of interaction modifies additivity in Murofushi \& Soneda (1993) and Grabisch (1996).

Statistical dependence is a different subject. It may be present if the assessments of preference by the different criteria are disturbed by random factors that jointly modify the results of the application of the different criteria. Such factors may be found in joint contamination of the generation or the collection of the evaluations of the alternatives. Interaction may be present, even if there is no such statistical dependence in the process of generating the measurements.

The difference between statistical dependence and interaction can be clearly seen in the case of sets of indicators of disjoint events in a probability space. Statistical dependence between them is always negative because the occurrence of any one of the events precludes the occurrence of the other. This does not happen to the concept of interaction, which involves a change of meaning of the indicators when the events are considered together, as the information on their joint nonoccurrence helps the decision maker to choose another alternative. We may rely on the observed data to decide on the presence of interaction between the criteria, but not using the same tools employed to decide on the presence of statistical dependence.

\subsection{The Shapley value}

A measure of the importance of a criterion in a capacity is given by its Shapley value (Shapley, 1953; Murofushi \& Soneda, 1993). 
The Shapley value of an element $c$ of a finite set $C$ for a capacity $\mu$ on $C$ estimates the increase in the capacity of an arbitrary subset of $C$ when $c$ is included in it. It is given by

$$
\operatorname{Shapley}(\mu, c)=\sum_{K \subset C \backslash\{C\}}[\#(C \backslash\{c\} \backslash K) ! \#(K) ! / \#(C) !][\mu(K\{c\})-\mu(K)],
$$

for \# denoting the function that associates to each set its cardinality and ! denoting the factorial. If $\mu$ is a probability, $K$ and $\{c\}$ being disjoint sets implies

$$
\mu(K \cup\{c\}-\mu(K)=\mu(\{c\}),
$$

so that the Shapley value of any criterion is equal to its probability.

If a criterion tends to present positive interaction with other criteria, its Shapley value will be higher than its individual capacity. The opposite will happen if it tends to interact negatively. Thus, how the Shapley value deviates from the capacity of the criterion measures the importance of its interactions.

\subsection{Determination of capacities}

In multicriteria decision analysis, if additivity can be assumed, the importance of the criteria can be given by a probability function in the space of criteria. A simple rule to determine such probability function, following the principle that the most important criteria for the decision maker are those ablest to select a single alternative as the best, consists in assigning to each criterion a probability proportional to the largest among the probabilities of preference that it assigns to the different alternatives. To satisfy the restriction of sum 1, these maxima will be divided by a constant of proportionality given by the probability of the maximum of the whole set of criteria. In the present case, by additivity, this constant is the sum of the probabilities of the individual criteria.

If additivity is not assumed, a rule is needed to generate, from the evaluations of the set of alternatives by the criteria, not a simple probability, but, with higher generality, a capacity. In that case, the principle of preference concentration leads us to start, as in the case of a probability, making the vector of capacities of the unitary sets proportional to the vector of maxima. The constraint of sum 1 for the values assigned to the unitary sets no longer holds. To bring the range to $[0,1]$, the capacity of any set, unitary or not, will still be obtained dividing the largest among the probabilities of preference assigned to the alternatives considering the criteria in that set by the largest preference by the set of all the criteria.

Thus, the principle of preference concentration will again, in the case of sets of more than one criterion, determine the capacities of the sets of criteria from the observed probabilities of preference according to each criterion. The interaction between two criteria will be measured by the increase or reduction in the maximum of the probabilities of preference if any one of the two criteria can be applied instead of only one of them.

This is extended from pairs of criteria to pairs of sets of any number of criteria. The comparison now will be between the values of the highest possible probability of preference by criteria of 
each set separately and considering the two sets together. The interaction will then be accessed by the difference between the probabilities of maximization of preference by any element of the union of the two sets and by an element of only one of them.

If there is negative interaction in the maximization of preference, the alternatives with maximal preference according to the two sets will not be the same preferred according to each of them separately. On the other hand, if there is positive interaction, the preference will be confirmed and the increase in the probability of preference by that alternative that maximizes preference will reflect that.

The increase in the probability of maximum preference measured in this way does not access in a balanced way the conflict between the positive interaction that would result in the confirmation of the preferred alternative by the two sets and the negative interaction that would bring any of the other alternatives with divergent evaluations to be the jointly preferred alternative. The higher possibility of change from one alternative to any other as the preferred one favors the assessment of negative interaction over the assessment of positive interaction. This explains the need to divide by the constant of proportionality given by the maximum when all the criteria are considered.

\subsubsection{Formal presentation of the algorithm for the determination of the capacity}

Summarizing what is described in the preceding subsection, the algorithm to generate the capacity of any subset $\left\{C_{j 1}, \ldots, C_{j s}\right\}$ of $s$ criteria of a set $C$ of $m$ criteria employed in the evaluation of a set of alternatives $\left\{A_{1}, \ldots, A_{n}\right\}$ consists in the steps described below with the aid of the following notation.

Let $P_{i j}$ denote the preference by alternative $A_{i}$ according to criterion $C_{j}$.

Let

$$
P_{i}\left(\left\{C_{j 1}, \ldots, C_{j s}\right\}\right)=1-\left(1-P_{i j 1}\right) \ldots\left(1-P_{i j s}\right)
$$

$P_{i}(\{C j 1, \ldots, C j s\})$ is the probability, under independence, of the evaluation of alternative $A_{i}$ presenting the highest evaluation for at least one of the $s$ criteria in the set $\left(C_{j 1}, \ldots, C_{j s}\right)$.

Let

$$
P\left(\left\{C_{j 1}, \ldots, C_{j s}\right\}\right)=\max _{i \in\{1, \ldots, n\}} P_{i}\left(\left\{C_{j 1}, \ldots, C_{j s}\right\}\right)
$$

The capacity of $\left\{C_{j 1}, \ldots, C_{j s}\right\}$ will be

$$
\text { Capacity }\left(\left\{C_{j 1}, \ldots, C_{j s}\right\}\right)=P\left(\left\{C_{j 1}, \ldots, C_{j s}\right\}\right) / P(C) .
$$

By definition, a capacity on $C$ is any function $\mu$ from the set of subsets of $C$ on $[0,1]$ such that

$$
\text { for every pair }\{A, B\} \text { of subsets of } C \text { with } A \subseteq B, \mu(A) \leq \mu(B) \text {. }
$$


Then to prove that Capacity is, in fact, a capacity on $C$, it is enough to show that, for every pair of sets of criteria $A$ and $B$ with $A \subseteq B$,

$$
\max _{i \in\{1, \ldots, n\}} P_{i}(A) \leq \max _{i \in\{1, \ldots, n\}} P_{i}(B) .
$$

But this will follow immediately from the fact that, for any alternative $i$, if

$$
A \subseteq B, P_{i}(A) \leq P_{i}(B)
$$

\subsection{The Choquet integral}

The joint preference for an alternative may be computed by a Choquet integral of the function in the set of criteria that relates to each criterion its preference for the alternative. The Choquet integral has been proposed by many authors as an aggregation operator, an adequate substitute to the weighted arithmetic mean to aggregate interacting criteria (Grabisch, 1996; Marichal, 2000; Labreuche \& Grabisch, 2006; Gomes et al., 2015).

The Choquet integral of a function $f$ defined on a set $C$ of $m$ elements $\left\{C_{1}, \ldots, C_{m}\right\}$ with respect to the capacity $\mu$ on $C$ is

$$
C_{\mu}(f)=\sum_{j=1}^{m}\left[f\left(C_{\pi(j)}\right)-f\left(C_{\pi(j-1)}\right)\right] \mu\left(\left\{C_{\pi(j)}, \ldots, C_{\pi(m)}\right\}\right),
$$

where $\pi$ is a permutation of $\{1, \ldots, m\}$ such that

$$
f\left(C_{\pi(1)}\right) \leq \cdots \leq f\left(C_{\pi(m)}\right)
$$

and

$$
f\left(C_{\pi(0)}\right)=0
$$

Equivalently,

$$
\left.C_{\mu}(f)=\sum_{j=1}^{m} f\left(C_{\pi(j)}\right)\left[\mu\left(\left\{C_{\pi(j)}, \ldots, C_{\pi(m)}\right\}\right)-\mu\left(\left\{C_{\pi(j+1)}\right), \ldots, C_{\pi(m)}\right\}\right)\right]
$$

with

$$
\mu\left(\left\{C_{\pi(m+1)}, C_{\mu(m)}\right\}\right)=0 .
$$

In Equation (12), it can be seen that the weight of the criterion according to which the alternative has its higher preference assessment is equal to its individual capacity, while the other criteria have their coefficients increased or decreased according to its higher or lower interaction with those assigning higher preference assessments to the alternative. Thus, the modeling of interaction in the capacity by the principle of preference concentration may combine with the use of the Choquet integral to magnify the score for those alternatives jointly preferred by different criteria. Alternatives with divergent evaluations by criteria with positive interaction have their evaluation 
by the Choquet integral reduced and those with concordant high evaluations by such criteria have their evaluation increased.

On the other hand, the Choquet integral reduces the influence of preferences of small value by criteria with negative interactions with the others. By this way, the concentration of preference on any alternative due to the increase of the capacity of isolated criteria will be eventually attenuated when the evaluations by the different criteria are combined by the Choquet integral.

Other simple properties of the Choquet integral are:

1. The Choquet integral is idempotent, i.e.,

$$
\text { for all } \mu \text {, if } f(x)=a \text { for all } x \text {, then } C_{\mu}(f)=a \text {; }
$$

2. The Choquet integral is nondecreasing, i.e.,

$$
\text { for all } \mu \text {, if } f_{1} \leq f_{2} \text {, then } C_{\mu}\left(f_{1}\right) \leq C_{\mu} f_{2} \text {; }
$$

3. The Choquet integral is always comprised between min and max.

4. The Choquet integral with respect to a probability coincides with the expected value.

5. The Choquet integral is stable under positive linear transformations.

6. All order statistics, in particular, min, max and median, can be obtained as Choquet integrals.

\subsection{Simplification of the Choquet integral}

Taking into account interactions between sets of any number of criteria may turn the interpretation of the results of the application of the Choquet integral too complex. To simplify, assumptions of $k$-additivity (Grabisch et al., 2008) may limit to a maximum $k$ the number of criteria that interact. A more direct simplification may be done by limiting the number of criteria effectively entering into the computation of the Choquet integral. This is obtained by assigning capacity 1 to any set of more than a small number $q$ of criteria and, for the sets of at most q criteria, employing, in the final standardization stage above described, instead of the division by the maximum of the preferences along all the criteria, the division by the maximum of the preferences by $q$ criteria. This implies assuming the absence of positive interaction between sets of more than $q$ criteria and results in considering, to compose the score of each alternative, only its $q$ or $q+1$ highest probabilities of being preferred by different criteria.

In the Choquet integral, an alternative of high preference according to a criterion with high positive interaction with the other criteria will have a higher score than if such criterion were negatively correlated with the others. Thus criteria with positive interactions are more important than their individual capacities imply and the inverse for criteria with dominantly negative interactions. The increase or reduction of importance due to this limitation to sets of small size may be evaluated by the variation of the Shapley value as different values of $q$ are employed. 


\subsection{Other Forms of Composition}

The approach above developed involves a few choices. Some of these choices are derived from the principle of preference concentration. Others, like the hypothesis of independence in the computation of the initial probabilities or the hypothesis implicit in the choice for the Choquet integral, are essentially derived from the intention to keep a balanced approach to the data. Different assumptions will lead to different paths and different results.

\subsubsection{Preference Dilution}

A psychological motivation contrary to preference concentration may prevail. A cautious decision maker, instead of preference concentration may wish to value the criteria in the opposite sense. This would be satisfied by assigning to each set of criteria a capacity proportional to the maximum of the probability of not presenting the highest evaluation by at least one of the criteria in the set.

This alternative capacity would be built by, after substituting, in Equation (3), $1-\pi_{k \in\{1, \ldots, s\}} P_{i j k}$ for $1-\left(1-P_{i j 1}\right) \ldots\left(1-P_{i j s}\right)$, following the same maximization and standardization steps above described.

A comparison of the results of the application of these two approaches may be used in a sensibility analysis. Other forms of composition may also be evaluated by the position of the results they generate compared to the results obtained applying the composition based on each of these two principles.

\subsubsection{Maximal Dependence}

The hypothesis of independence in the formulations above developed follows from the assumption that suitable controls rule the process of data collection. Independence is recommended to pursue in the design and application of the criteria, as the data collected are naturally read as independent. If there is any reason to believe in the presence of a particular form of statistical dependence, the computation may take it into account.

In practice, the presence of more complex forms of dependence must be precisely identified to justify modifications in the treatment of the observed assessments. In fact, the hypothesis of independence, by giving equal relevance to the assessments by all the criteria, tends to provide a robust approximation. On the contrary, a simpler hypothesis of maximal dependence, for instance, would lead to disregard the numerical values of the smaller $P_{i j l}$. Whenever this hypothesis might be accepted, it would justify replacement, in Equation (3), of $1-\left(1-P_{i j 1}\right) \ldots\left(1-P_{i j s}\right)$ by $\max _{1 \in\{1, \ldots, n\}} P_{i j l}$. 


\subsubsection{Generalized Additive Independence}

After determining the capacities, another approach to the composition of the preferences according to the different criteria that would take into account the interactions between the criteria but would not explore the preference concentration implicit in the use of the Choquet integral might be derived from application of the assumption of GAI.

The absence of interaction implies that the global preference between any two alternatives is determined by their preferences according to the isolated criteria. This concept can be generalized to sets of criteria. The property of GAI holds for a family of sets of criteria $C_{Z 1}, \ldots, C_{Z g}$, not necessarily disjoint, if and only if the global preference between any two alternatives is determined by the preferences according to these sets. In the case of GAI, it can be shown (Bacchus $\&$ Grove, 1995) that the global preference for alternative $i$ with respect to the capacity $\mu$ is given by

$$
G_{\mu}(i)=\sum_{h=1}^{g} P_{i}\left(C_{Z h}\right) \mu\left(C_{Z h}\right),
$$

for all family of sets of criteria $\left\{C_{Z 1}, \ldots, C_{Z g}\right\}$ GAI with respect to $\mu$ such that

$$
\cup_{h=1}^{g} C_{Z h}=C
$$

It may happen that the decision maker does not wish to benefit from the concentration feature of the Choquet integral. Then, once established GAI for sets of at most q criteria, the procedures above proposed may be used to derive the capacity and the probabilities of preference for each alternative by sets of that size and the global preferences can be obtained applying Equation (16) with a relatively small set of summands.

\section{APPLICATIONS}

This section shows how the principle of preference concentration may be applied in different decision problems. The first application is limited to the assignment of importance to criteria and out of the context of criteria composition. It is designed to show only how the psychogical motivation for preference concentration works and that this motivation keeps coherence with the practice in a special case. The application of the principle to the choice between three criteria is employed in the context of the Ellsberg problem. The preference to decide by comparing maximum expected value, which is the criterion indicated by preference concentration, leads to the behavior observed in practice.

The main objective of the other two applications is to demonstrate the viability of the three steps procedure above presented and the consistency of the results obtained in different contexts. The second centers in the construction of a capacity in a numerical example of choice between two and three alternatives according to three criteria. The first step is simplified, with the probabilities of preference used in the probabilistic composition assumed previously known. The second step, of derivation of the capacity, is developed in detail. In the third step, of the final composition of the preferences taking into account the interactions measured in the previous step, it is discussed 
how the Shapley value may be employed to evaluate the possibility of simplifying the computation of the Choquet integral by reducing the size of the sets for which the possibility of positive interaction between criteria is allowed.

The third application is to a real life situation of ranking students on the basis of their grades. In this application, the first step is completely described, with the use of the triangular distribution in the modeling of the probabilities of being the best according to each criterion. Interesting to notice in this application is the proximity of the results obtained to those obtained by Kojadinovic (2004) building the capacity on the basis or the principle of entropy maximization.

The third application is to a real life situation of ranking students on the basis of their grades. In this application, the first step is completely described, with the use of the triangular distribution in the modeling of the probabilities of being the best according to each criterion. Interesting to notice in this application is the proximity of the results obtained to those obtained by Kojadinovic (2004) building the capacity on the basis or the principle of entropy maximization.

\subsection{Application of the Principle of Preference Concentration to the Ellsberg Problem}

The aversion to uncertainty in decision-making represented by the principle of preference concentration leads to the assignment of higher importance to the criteria that more clearly highlight the best alternatives. In this first application, it is applied to explain the preference for one among three criteria that may be employed in the choice known as the paradox of Ellsberg (Ellsberg, 1961; Aerts et al., 2014).

The problem of Ellsberg is a simple problem for which the decision makers give contradictory answers. It can be formulated with 3 balls in an urn. One of the balls is red and the other two are equally likely to be blue or yellow. Asked to choose the color of the ball that will be drawn from the urn, the majority chooses the first. But, if people are given the right to bet on two different colors, the preference is for the pair formed by the other two. This change in the proposition of the problem with the addition of a second color to the first option can be seen as the presentation of the same options in the inverse form, as the choice of one color is equivalent to the rejection of the other two. But it can be seen that the two problems are not the same if due attention is given to the importance that has for the decision maker the possibility of being able to discern the best option.

The two problems may be put in terms of finding the best criterion, with the criteria formulated as functions of the expected value of each option under the admitted hypotheses about the distribution of colors.

The principle of preference concentration leads to value a criterion by its ability to strengthen the conviction about which is the best option. It is applied here to compare three criteria, based respectively in evaluating the three options by the minimum $\left(C_{1}\right)$, the mean $\left(C_{2}\right)$ and the maximum $\left(C_{3}\right)$ expected value, calculated for the three possible probability distributions in the state space of the experiment of drawing one of the three balls from the urn. 
These three probability distributions, with equal prior probabilities, are:

$H_{1}$ there are, in the urn, two yellow balls;

$\mathrm{H}_{2}$ there are, in the urn, two blue balls;

$H_{3}$ there are, in the urn, a blue and a yellow ball.

In the first problem, the choice is between three events: $A_{1}:$ a red ball is drawn, $A_{2}:$ a blue ball is drawn, $A_{3}$ : a yellow ball is drawn. In the second, between the three complementary events: $A_{4}$ : a red ball is not drawn, $A_{5}$ : a blue ball is not drawn, $A_{6}$ : a yellow ball is not drawn.

$A_{2}$ and $A_{3}$ - and consequently, $A_{5}$ and $A_{6}$ - are clearly equivalent.

Then the two problems are determined by the conditional probabilities:

$$
\begin{aligned}
& P\left(A_{1} \mid H_{1}\right)=P\left(A_{1} \mid H_{2}\right)=P\left(A_{1} \mid H_{3}\right)=1 / 3, \\
& P\left(A_{2} \mid H_{1}\right)=0, P\left(A_{2} \mid H_{2}\right)=2 / 3, P\left(A_{2} \mid H_{3}\right)=1 / 3 .
\end{aligned}
$$

Below follows the evaluations of the three options by the three criteria.

$$
\begin{aligned}
& C_{1} \text { - minimum expected value: } \\
& C_{1}\left(A_{1}\right)=P\left(A_{1} \mid H_{1}\right)=P\left(A_{1} \mid H_{2}\right)=P\left(A_{1} \mid H_{3}\right)=1 / 3 \text { and } \\
& C_{1}\left(A_{2}\right)=C_{1}\left(A_{3}\right)=P\left(A_{2} \mid H_{1}\right)=0 \\
& C_{1}\left(A_{4}\right)=1-C_{1}\left(A_{1}\right)=2 / 3 \text { and } \\
& C_{1}\left(A_{5}\right)=C_{1}\left(A_{6}\right)=1-P\left(A_{2} \mid H_{2}\right)=1 / 3 .
\end{aligned}
$$

$C_{2}$ mean expected value:

$$
\begin{aligned}
& C_{2}\left(A_{1}\right)=\sum_{i} P\left(A_{1} \mid H i\right)^{*} P(H i)=1 / 3 . \\
& C_{2}\left(A_{3}\right)=C_{2}\left(A_{2}\right)=\sum_{i} P\left(A_{2} \mid H_{i}\right)^{*} P\left(H_{i}\right) \\
& \quad=0^{*} 1 / 3+2 / 3^{*} 1 / 3+1 / 3^{*} 1 / 3=1 / 3 . \\
& C_{2}\left(A_{4}\right)=1-C_{2}\left(A_{1}\right)=2 / 3 . \\
& C_{2}\left(A_{6}\right)=C_{2}\left(A_{5}\right)=1-C_{2}\left(A_{2}\right)=2 / 3 .
\end{aligned}
$$

$C_{3}$ maximum expected value:

$$
\begin{aligned}
& C_{3}\left(A_{1}\right)=P\left(A_{1} \mid H_{1}\right)=P\left(A_{1} \mid H_{2}\right)=P\left(A_{1} \mid H_{3}\right)=1 / 3 . \\
& C_{3}\left(A_{2}\right)=C_{3}\left(A_{3}\right)=P\left(A_{2} \mid H_{2}\right)=2 / 3 . \\
& C_{3}\left(A_{4}\right)=1-C_{3}\left(A_{1}\right)=2 / 3 . \\
& C_{3}\left(A_{5}\right)=C_{3}\left(A_{6}\right)=1-P\left(A_{2} \mid H_{1}\right)=1 .
\end{aligned}
$$


The application of the principle of preference concentration leads to discarding $C_{2}$, for both problems, because it presents a tie between the three options. $C_{3}$ will also be less valued than $C_{1}$ because it leads to a tie between two preferred options, while $C_{1}$ points to a best option. This preferred option is that one preferred by the majority of people facing the Ellsberg problem.

Taking the expected gain as the basis to decide, an infinite continuum of criteria exists. The comparison of the three here considered is enough to show how the motivation for preference concentration favors the criteria that result in the choices observed in practice.

Similar problems, formulated in a context where asymmetry between gains and losses is conceivable, admit explanations for the preference to decide by maximizing the minimum expected value based on the prevalence of aversion to losses against attraction to gains (Kahneman \& Tversky, 1984; McFadden et al., 1999). But in the Ellsberg urn gains and losses are interchangeable.

\subsection{Numerical Example}

For a simple numerical example, let us consider a case of only two alternatives. Three criteria, say, Severity $(S)$, Occurrence $(O)$ and Undetectability $(D)$, are applied, and the probabilities of preference are those in Table 1.

Table 1 - Probabilities of preference for two alternatives by three criteria.

\begin{tabular}{c|c|c|c}
\hline & $S$ & $O$ & $D$ \\
\hline Alternative1 & 0.6 & 0.2 & 0.6 \\
\hline Alternative2 & 0.4 & 0.8 & 0.4 \\
\hline
\end{tabular}

If the composition of the preferences is made by weighted average with the weights determined by the principle of preference concentration, these weights, proportional to the maxima $0.6,0.8$ and 0.6 , will be $0.3,0.4$ and 0.3 . The scores will then be 0.44 for Alternative 1 and 0.56 for Alternative2, and Alternative2 will be chosen.

To build a capacity on the space of criteria that takes into account the possible interactions, the first step is to determine the probabilities of preference by at least one of the elements of the sets of two and three criteria. These probabilities are given in Table 2.

Table 2 - Probabilities of preference by at least one of the criteria.

\begin{tabular}{c|c|c|c|c}
\hline & $\{S, O\}$ & $\{S, D\}$ & $\{O, D\}$ & $\{S, O, D\}$ \\
\hline Alternative1 & $1-0.4 * 0.8=0.68$ & $1-0.4 * 0.4=.0 .84$ & $1-0.8 * 0.4=0.68$ & $1-.4 * .8 * .4=0.872$ \\
\hline Alternative2 & $1-0.6 * 0.2=0.88$ & $1-0.6 * 0.6=0.64$ & $1-0.2 * 0.6=0.88$ & $1-.6 * .2 * .6=0.928$ \\
\hline
\end{tabular}

Table 3 presents the capacities derived from the largest probabilities in Table 2, for the values of $q$ of 2 and 3. 
Table 3 - Capacities for different values of $q$ derived from preferences for two alternatives.

\begin{tabular}{l|c|c|c|c|c|c|c}
\hline & $S$ & $O$ & $D$ & $\{S, O\}$ & $\{S, D\}$ & $\{O, D\}$ & $\{S, O, D\}$ \\
\hline$q=3$ Maxima & 0.6 & 0.8 & 0.6 & 0.88 & 0.84 & 0.88 & 0.928 \\
\hline$q=3$ Capacities & 0.647 & 0.862 & 0.647 & 0.948 & 0.905 & 0.948 & 1 \\
\hline$q=2$ Maxima & 0.6 & 0.8 & 0.6 & 0.88 & 0.84 & 0.88 & \\
\hline$q=2$ Capacities & 0.682 & 0.909 & 0.682 & 1 & 0.955 & 1 & 1 \\
\hline
\end{tabular}

The final scores obtained for the two alternatives applying the Choquet integral with respect to the capacities in Table 3 are, for Alternative1, 0.575 and 0.582 for $q=3$ and 2, respectively, and, for Alternative2, 0.750 for $q=3$ and 0.764 for $q=2$. Thus, the decision is still the same reached under the assumption of null interaction, as the composed score for Alternative2 is always higher than the composed score for Alternative1, whatever the choice of $q$.

The Shapley values for the criteria are $0.302,0.396$ and 0.302 , respectively, for $q=3$, and 0.288 , 0.424 and 0.288 , respectively, for $q=2$. These values are close to the probabilities $0.3,0.4$ and 0.3 , and, considerably lower when compared to the individual capacities in Table 2. They are similar for $q=2$ and $q=3$, signaling that the interactions between pairs of criteria are more important than the interactions of each criterion with the pair of two other criteria.

It may be interesting to notice that this result is due to the preference for Alternative 2 by Occurrence being relatively higher than the preference for Alternative1 by Severity or by Undetectability. In fact, if, for instance, the value of the probability of preference for the preferred alternative is the same for the three criteria, then the joint preference would go for Alternative1.

In that case, even if the preference assigned to the preferred alternative is close to the other, the principle of preference concentration would turn the final preference to Alternative1, because it is preferred by two criteria. For the values of 0.501 and 0.499 , the composed scores will differ by 0.0001 , for $q=3$, and 0.007 , for $q=2$. This difference will gradually increase as the differences between the initial probabilities of preference increase. For preferences of $5 / 6$ for the preferred alternative and 1/6 for the other, the final scores for Alternative1 will be 0.832 for $q=3$ and 0.611 for $q=2$.

Let us consider now the addition of a third alternative, Alternative3, which absorbs part of the preference for the preferred alternative according to each criterion. If it absorbs also part of the preference for the other alternative and, consequently, the maximal probabilities of preference do not change, no considerable change will appear in the Choquet integrals. If the preference for Alternative1 is reduced from 0.6 to 0.5 in Severity and Undetectability, and the preference for Alternative 2 by Occurrence is reduced from 0.8 to 0.6 , with the transference of 0.1 and 0.2 , respectively, to Alternative3, the final scores still favor Alternative2. Nevertheless, a change that shows the importance kept by secondary preferences is obtained if the probabilities of 0.4 and 0.1 of the second and third positions according to Severity and Undetectability are switched between Alternative 2 and Alternative3. Table 4 presents the resulting capacities for this case. 
Table 4 - Capacities for different values of $q$ derived from preferences for three alternatives.

\begin{tabular}{l|c|c|c|c|c|c|c}
\hline & $S$ & $O$ & $D$ & $\{S, O\}$ & $\{S, D\}$ & $\{O, D\}$ & $\{S, O, D\}$ \\
\hline$q=3$ Maxima & 0.5 & 0.6 & 0.5 & 0.64 & 0.75 & 0.64 & 0.8 \\
\hline$q=3$ Capacities & 0.625 & 0.75 & 0.625 & 0.8 & 0.9375 & 0.8 & 1 \\
\hline$q=2$ Maxima & 0.5 & 0.6 & 0.5 & 0.64 & 0.75 & 0.64 & \\
\hline$q=2$ Capacities & 0.667 & 0.8 & 0.667 & 0.853 & 1 & 0.853 & 1 \\
\hline
\end{tabular}

With respect to Table 2, Table 4 shows not only a reduction in the relative importance of Occurrence when the criteria are considered alone but, also, in the capacities of the pairs of criteria, with a considerable gain for the pair $\{$ Severity, Undetectability $\}$.

Assuming additivity, the weights will be $0.3125,0.375$ and 0.325 , for Severity, Occurrence and Undetectability with scores of $0.3875,0.2875$ and 0.325 for Alternative 1 , Alternative 2 and Alternative3, respectively. The Choquet integrals with respect to the capacities in Table 4 will give a small advantage to Alternative1 for $q=3$, while a tie happens for $q=2$. The scores will be $0.48125,0.475$ and 0.3875 , for $q=3$, and $0.5,0.5$ and 0.4 for $q=2$. For $q=3$ and $q=2$, the Shapley values are again very similar, with approximate values of $0.335,0.330$ and 0.335 , confirming the reduced effect of disregarding the interaction of more than two criteria.

\subsection{Comparison to entropy maximization}

The principle of preference concentration differs from that principle followed in the approach proposed by Kojadinovic (2004), which measures the capacity by the information, in the sense of Shannon (1948) or Rényi et al. (1960), contained in the distribution of preferences. This information is estimated by Kojadinovic (2004) by means of the relation between the entropy in the matrix of the assessments of preference according to the criteria in the set and the entropy in the matrix of all the available assessments of preference. This makes the capacity of a set larger as the distribution of the evaluations according to the criteria in the set spreads more uniformly along the alternatives. Especially, in the case of unitary sets, the capacity of a single criterion increases with the uniformity in the assessments of preference according to the criterion. The principle of preference concentration, differently, leads to assign a larger capacity to those criteria with a higher ability to discriminate the best alternative.

A problem of ranking by the Choquet integral with respect to a capacity whose interaction between criteria is determined on the basis of the information measure contained in the matrix of preference distributions is studied by Kojadinovic (2004). The performances of 89 students in 5 subjects, English (Eng), Computer Science (Com), Algebra (Alg), Analysis (Ana) and Physics (Phy), are compared. The initial figures are the grades of the students, given by integers in a range from 0 to 20 . These grades are linearly brought to values between 0 and 1, translating to 0 the lowest and to 1 the highest observed value in each subject. The same data are here analyzed, treating these values as modes of triangular distributions with extremes at 0 and 1 . 
First, composition by weighted average, with the weights determined by preference concentration assuming additivity, was performed. Then the capacity was derived and scores composed by the Choquet integral were computed. Both forms of composition selected as the best joint performance that of Student 41 . This is the same result obtained applying the Choquet integral with respect to the capacity built following the rule of maximization of entropy measured by Shannon information.

The probabilities of maximization of the grades in each subject and the composition by the Choquet integral were obtained using the software R (Venables WN \& the R Development Core Team, 2015). The kappalab package (Grabisch et al., 2008) of $\mathrm{R}$ was used in the computation of the Choquet integrals.

Table 5 shows the capacities of the 30 sets of criteria of 1 to 4 elements derived from the probabilities of maximization. The values in Table 5 were obtained for $q=5$. Capacities and final scores close to those were obtained for the other possible values of $q$. The rank correlation coefficients of the scores by the Choquet integral with the capacity obtained with $q=5$ and those obtained with other values of $q$ are above 0.99 .

Table 5 - Capacity reflecting preference concentration for criteria based on grades.

\begin{tabular}{l|c|c|c|c|c|c|c}
\hline Eng & 0.39 & Eng-Com & 0.48 & Eng-Com-Alg & 0.62 & Eng-Com-Alg-Ana & 0.63 \\
\hline Com & 0.42 & Eng-Alg & 0.52 & Eng-Com-Ana & 0.50 & Eng-Com-Alg-Phy & 0.99 \\
\hline Alg & 0.43 & Eng-Ana & 0.44 & Eng-Com-Phy & 0.63 & Eng-Com-Ana-Phy & 0.64 \\
\hline Ana & 0.37 & Eng-Phy & 0.51 & Eng-Alg-Ana & 0.55 & Eng-Alg-Ana-Phy & 0.90 \\
\hline Phy & 0.45 & Com-Alg & 0.55 & Eng-Alg-Phy & 0.89 & Com-Alg-Ana-Phy & 0.94 \\
\hline & & Com-Ana & 0.45 & Eng-Ana-Phy & 0.52 & & \\
\hline & & Com-Phy & 0.57 & Com-Alg-Ana & 0.56 & & \\
\hline & & Alg-Ana & 0.45 & Com-Alg-Phy & 0.93 & & \\
\hline & & Alg-Phy & 0.83 & Com-Ana-Phy & 0.58 & & \\
\hline & & Ana-Phy & 0.46 & Alg-Ana-Phy & 0.84 & &
\end{tabular}

The concordance between the decision by the Choquet integral with respect to the capacity determined by maximizing entropy and by preference concentration may be explained by the occurrence in this kind of data of high consistency in the performance in the different school subjects for most students. This reduces the variability of the entropy along the matrices.

Some differences can nevertheless be noticed. For instance, although there is no large difference between the capacities of the unitary sets, all around 0.38 in the capacity determined by Shannon entropy and ranging from 0.37 to 0.43 in the capacity determined by the principle of preference concentration, there is a considerable difference between the vectors of Shapley values, presented in Table 6. While the entropy maximization brings again considerably uniform Shapley values, the principle of preference concentration applied to the probabilities of maximization produces a capacity with higher Shapley values for the subjects of Algebra and Physics. The increase in the importance given to the sets where these two criteria appear together can also be noticed in 
Table 5. The difference in the Shapley values increases the chance of different compositions rules being able to lead to different results.

Table 6 - Shapley values for school subject grades.

\begin{tabular}{l|c|c|c|c|c}
\hline & Eng & Com & Alg & Ana & Phy \\
\hline Concentration & 0.13 & 0.17 & 0.30 & 0.09 & 0.31 \\
\hline Entropy & 0.21 & 0.21 & 0.19 & 0.19 & 0.19 \\
\hline
\end{tabular}

\section{FINAL COMMENTS}

The principle of preference concentration explains the decision maker behavior in different situations. The adoption of this principle provides a new angle from which information about preferences can be collected. Its application to multicriteria decision analysis provides support for a framework to combine preferences by Choquet integrals based on the automatic construction of capacities on the set of criteria.

The resulting composition by the Choquet integral with respect to capacities determined by unsupervised algorithms highlights those alternatives preferred by the criteria considered, avoiding externally interfering with the modeling of the relations between them. Besides, it propitiates a better understanding of the relative importance of each criterion for setting the preferences, both by the capacity allocated to the criteria isolated and by their Shapley values.

The prevalence of this principle must face the test of reality in varied situations along time. The approach here developed to multicriteria decision aid must be compared to methodologies based on other forms of determining weights and of measuring interactions, and especially to other forms of composition that may be developed to avoid the difficulties of finding the right weights and interactions.

This approach overrides a classical view that the decision maker might act successively, first objectively deciding on weights for the criteria and, after that, independently applying each criterion to evaluate the alternatives. It considers that, when the decision maker applies the criteria, the probabilistic preferences among the alternatives inform also on subjective preferences among the criteria. For instance, if a criterion has low importance for the decision maker, the variance in the probabilistic preferences for each alternative according to this criterion may place the alternatives closer to each other than the probabilistic preferences according to other criteria that the decision maker evaluates as more important.

Developments are welcome in the management of the application of the approach here proposed to different practical decision problems. For instance, forms of computation of the initial joint preferences under different assumptions and determination of numerical bounds for statistical dependence may be useful.

Further developments may be the comparison of the application of the principle of preference concentration with the application of other principles that may drive the assignment of impor- 
tance to the criteria, like that of preference dilution, and the comparison of the Choquet integral with other composition rules, like that based on generalized additive independence. It would be of practical interest evaluate the suitability of the application of different principles and of different composition rules to different real conditions of interaction.

This work is based on the belief that the information about the relative importance of multiple criteria derived from data generated when the criteria are applied may be more reliable than that obtained when preferences about the criteria are elicited in abstract comparisons. This is a theoretical contention that should also be subject to further investigation.

\section{ACKNOWLEDGEMENTS}

The authors are grateful to three Pesquisa Operacional anonymous reviewers for their helpful comments and suggestions.

\section{REFERENCES}

[1] Aerts D, Sozzo S \& TAPIA J. 2014. Identifying quantum structures in the Ellsberg paradox. International Journal of Theoretical Physics, 53(10): 3666-3682.

[2] Aires RFdF \& Ferreira L. 2018. The Rank Reversal Problem in Multi-Criteria Decision Making: A Literature Review. Pesquisa Operacional, 38(2): 331-362.

[3] Angilella S, Corrente S \& Greco S. 2015. Stochastic multiobjective acceptability analysis for the Choquet integral preference model and the scale construction problem. European Journal of Operational Research, 240(1): 172-182.

[4] Bacchus F \& Grove A. 1995. Graphical models for preference and utility. In: Proceedings of the Eleventh conference on Uncertainty in artificial intelligence. pp. 3-10. Morgan Kaufmann Publishers Inc.

[5] Benabbou N, Perny P \& Viappiani P. 2017. Incremental elicitation of Choquet capacities for multicriteria choice, ranking and sorting problems. Artificial Intelligence, 246: $152-181$.

[6] BORN M. 1954. Statistical interpretation of Quantum Analysis. Science, 122: 675-679.

[7] CAMERER CF. 1987. Do biases in probability judgment matter in markets? Experimental evidence. American Economic Review, 77(5): 981-997.

[8] Choquet G. 1953. Theory of capacities. Annales de l' Institutc Fourier, 5: 131-295.

[9] Colorni A \& TSOUKiÀs A. 2018. What is a decision problem: Designing alternatives. In: Matsatsinis N \& GRigoroudis E (Ed.), Preference Disaggregation in Multiple Criteria Decision Analysis: Essays in Honor of Yannis Siskos. Cham: Springer. 
[10] DANE E \& PRATT MG. 2007. Exploring intuition and its role in managerial decision making. Academy of Management Review, 32(1): 33-54.

[11] DoYLE JR. 1995. Multiattribute choice for the lazy decision maker: Let the alternatives decide! Organizational Behavior and Human Decision Processes, 62(1): 87-100.

[12] Ellsberg D. 1961. Risk, ambiguity, and the Savage axioms. Quarterly Journal of Economics, 75(4): 643-669.

[13] Eyvindson K, Hujala T, Kurttila M \& Kangas A. 2015. Interactive preference elicitation incorporating a priori and a posteriori methods. Annals of Operations Research, 232(1): 99-113.

[14] FISHBURn PC. 1967. Additive Utilities with Incomplete Product Set: Applications to Priorities and Assignments. Baltimore: ORSA. 537-542 pp.

[15] FISHBURN PC. 1970. Utility theory for decision making. New York: Wiley.

[16] Garcia PAA \& SAnT'AnNA AP. 2015. Vendor and logistics provider selection in the construction sector: A probabilistic preferences composition approach. Pesquisa Operacional, 35(2): 363-375.

[17] Gilovich T, Griffin D \& Kahneman D. 2002. Heuristics and biases: The psychology of intuitive judgment. Cambridge: Cambridge University Press.

[18] Gomes LFAM, Machado MAS \& RANGel LAD. 2015. The multiple choice problem with interactions between criteria. Pesquisa Operacional, 35(3): 523-537.

[19] Grabisch M. 1996. The representation of importance and interaction of features by fuzzy measure. Pattern Recognition Letters, 17(6): 567-575.

[20] Grabisch M, Kojadinovic I \& Meyer P. 2008. A review of methods for capacity identification in Choquet integral based multi-attribute utility theory: Applications of the Kappalab R package. European Journal of Operational Research, 186(2): 766-785.

[21] Hammond JS, Keeney RL \& RAIfFA H. 2015. Smart choices: A practical guide to making better decisions. New York: Harvard Business Review Press.

[22] Hwang CL \& Yoon K. 1981. Multiple Attribute Decision Making Methods and Applications. New York: Springer.

[23] Kahneman D \& Tversky A. 1984. Choices, Values, and Frames. American Psychologist, 39(4): 341-350.

[24] Keeney RL \& Raiffa H. 1976. Decisions with Multiple Objectives. New York: Wiley.

[25] Khrennikova P \& Haven E. 2017. Voters' Preferences in a Quantum Framework. In: Haven E \& KhreniKova A (Eds.), The Palgrave Handbook of Quantum Models in Social Science. London: Palgrave MacMillan. 
[26] KoJADinOVic I. 2004. Unsupervised aggregation by the choquet integral based on entropy functionals: Application to the evaluation of students. In: TORRA V \& NARRUKAWA Y (Eds.), Modeling Decisions for artificial Intelligence, LNCS 3131. pp. 163-174. Heidelberg: Springer.

[27] Kong F. 2011. Rank reversal and rank preservation in TOPSIS. Advanced Materials Research, 204-210: 36-41.

[28] Labreuche C \& Grabisch M. 2006. Generalized Choquet-like aggregation functions for handling bipolar scales. European Journal of Operational Research, 172(3): 931-955.

[29] Labreuche C \& GRABISCh M. 2013. Use of the GAI model in multi-criteria decision making: inconsistency handling, interpretation. In: Proceedings of the International Conference of the Euro Society for Fuzzy Logic and Technology.

[30] Lootsma FA. 1996. A model for the relative importance of the criteria in the multiplicative AHP and SMART. European Journal of Operational Research, 94(3): 467-476.

[31] LuCE RD. 1959. Individual choice behavior: A theoretical analysis. New York: Wiley.

[32] Marichal JL. 2000. An axiomatic approach of the discrete Choquet integral as a tool to aggregate interacting criteria. IEEE Transactions on Fuzzy Systems, 8(6): 800-807.

[33] May JH, Shang J, TJader YC \& VArgas LG. 2013. A new methodology for sensitivity and stability analysis of analytic network models. European Journal of Operational Research, 224(1): 180-188.

[34] McFAdDen D, MAChina MJ \& BARON J. 1999. Rationality for economists? Journal of Risk and Uncertainty, 19(1-3): 73-105.

[35] Meyer P \& Olteanu AL. 2017. Integrating large positive and negative performance differences into multicriteria majority-rule sorting models. Computers \& Operations Research, 81: 216-230.

[36] Mimović P, Stanković J \& Milić VJ. 2015. Decision-making under uncertainty-the integrated approach of the AHP and Bayesian analysis. Economic Research-Ekonomska Istraživanja, 28(1): 868-877.

[37] Moreno-Jiménez JM, Salvador M, Gargallo P \& Altuzarra A. 2016. Systemic decision making in AHP: a Bayesian approach. Annals of Operations Research, 245(1-2): 261-284.

[38] Murofushi T \& Soneda S. 1993. Techniques for reading fuzzy measures (III): interaction index. In: Proceedings of the 9th Fuzzy Systems Symposium. pp. 693-696. Sapporo, Japan. 
[39] Plotnitsky A. 2009. Epistemology and Probability. Bohr, Heisenberg, Schrödinger, and the Nature of Quantum-Theoretical Thinking. Heidelberg: Springer.

[40] RÉNYi A ET AL. 1960. On measures of entropy and information. In: Proceedings of the Fourth Berkeley Symposium on Mathematical Statistics and Probability. pp. 547-561.

[41] Rowley HV, Geschke A \& Lenzen M. 2015. A practical approach for estimating weights of interacting criteria from profile sets. Fuzzy Sets and Systems, 272C: 70-88.

[42] SaAty TL. 1980. The Analytic Hierarchy Process. New York: McGraw Hill.

[43] SAATy TL. 1996. Decision making with dependence and feedback: The analytic network process. Pittsburgh: RWS Publications.

[44] SAATY TL \& BRAdy C. 2009. The Encyclicon, volume 2: A Dictionary of Complex Decisions using the Analytic Network Process. Pittsburgh: RWS Publication.

[45] Sant'Anna AP. 2010. Probabilistic composition of criteria for schedule monitoring. Pesquisa Operacional, 30(3): 751-767.

[46] SAnt'AnNA AP. 2015. Probabilistic composition of preferences, theory and applications. Heidelberg: Springer.

[47] Shannon CE. 1948. A mathematical theory of communication. Bell System Technical Journal, 27(3): 379-423.

[48] Shapley LS. 1953. A value for n-person games. In: KuHn HW \& TuCKER AW (Eds.), Contributions to the Theory of Games, Vol. II. Annals of Mathematics Studies, 28. pp. 307-317. Princeton: Princeton University Press.

[49] Simos J. 1974. L'évaluation environnementale: Un processus cognitif négocié. Ph.D. thesis. DGF-EPFL. Lausanne.

[50] Sugeno M. 1974. Theory of fuzzy integrals and its applications. Ph.D. thesis. Tokyo Institute of Technology. Tokyo.

[51] Venables WN SDM \& the R Development Core Team. 2015. An Introduction to R. Available at: https://cran.r-project.org/doc/manuals/R-intro.pdf.

[52] Vinogradova I, Podvezko V \& Zavadskas E. 2018. The recalculation of the weights of criteria in MCDM methods using the bayes approach. Symmetry, 10(6): 205. 